Staff for Operations and Plans, Department of the Army

Dawn Elizabeth Scalici, Intelligence Officer (Military), Office of Soviet Analysis, Strategic Forces Division, Central Intelligence Agency

Charles R. Taylor, Intelligence Analyst, Defense Intelligence Agency

Ralph B. Tildon, Jr., Senior Current Intellgence Officer, Central Intelligence Agency

Louis W. Weber, Major, PoliticoMilitary Staff Officer, Office of Deputy Chief of Staff for Operations and Plans, Department of the Army

Barry A. Zulauf, Intelligence Research Analyst, Office of Naval Intelligence, Department of the Navy

\section{Report of the Treasurer of the American Political Science Association, 1991-92}

\section{James A. Caporaso, University of Washington}

In the December 1991 issue of PS, former treasurer David Brady was able to report that the APSA was in good financial health. Since that time, the Association has continued to do well, both in terms of its annual budgets and its long-term investments.

Table 1 presents a balance sheet for all Association funds, including the General Operating Fund, the Trust and Development Fund, and Endowed Program Funds. Total funds of the Association increased from $\$ 3,604,864(1990)$ to $\$ 8,685,705$ (1991), largely due to the endowment of the Congressional Fellowship Program by MCI Communications Corporation, to $\$ 9,125,023$ (1992). We had a budget surplus of $\$ 74,000$ for fiscal year 1991-92 and our investment funds continue to grow (figures provided below).

To place our budget in perspective, Table 2 presents income and expendi- ture data from fiscal year 1979-80 to 1991-92. We ran a surplus in every year, last year being no exception. Expenditures increased by $2.2 \%$, a figure well below the average change since at least $1979-80$.

The continued growth and financial health of the Association depend on both prudent budgetary manage-

TABLE 2.

APSA Budget: A Multi-Year Perspective

\begin{tabular}{crrrr}
\hline Year & Income & Expenditures & $\begin{array}{c}\text { Surplus }+ \\
\text { Deficit }\end{array}$ & $\begin{array}{c}\text { Annual } \\
\text { Expenditure } \\
\text { Change (\%) }\end{array}$ \\
\hline $1979-80$ & $\$ 930,157$ & $\$ 929,857$ & +300 & +6.2 \\
$1980-81$ & $1,007,675$ & 977,328 & $+30,347$ & +5.1 \\
$1981-82$ & $1,117,701$ & $1,043,255$ & $+74,446$ & +6.7 \\
$1982-83$ & $1,202,078$ & $1,094,415$ & $+107,663$ & +4.9 \\
$1983-84$ & $1,323,074$ & $1,247,529$ & $+75,545$ & +14.0 \\
$1984-85$ & $1,413,078$ & $1,353,330$ & $+59,739$ & +6.5 \\
$1985-86$ & $1,505,224$ & $1,453,248$ & $+51,976$ & +3.1 \\
$1986-87$ & $1,584,945$ & $1,449,638$ & $+85,307$ & +4.2 \\
$1987-88$ & $1,637,637$ & $1,563,252$ & $+74,385$ & +10.7 \\
$1988-89$ & $1,847,152$ & $1,731,251$ & $+115,901$ & +14.0 \\
$1989-90$ & $1,891,773$ & $1,871,305$ & $+20,464$ & +2.2 \\
$1990-91$ & $2,153,800$ & $2,133,524$ & $+24,594$ & $+74,300$ \\
$1991-92$ & $2,254,844$ & $2,180,544$ & +74 & \\
\hline
\end{tabular}

TABLE 3.

Portfolio Summary, Trust \& Development Fund, June 30, 1992

\begin{tabular}{|c|c|c|c|c|c|}
\hline Security Type & Cost Value & Market Value & $\begin{array}{c}\% \\
\text { Assets }\end{array}$ & $\begin{array}{c}\text { Current } \\
\text { Yield }\end{array}$ & $\begin{array}{l}\text { Estimated } \\
\text { Annual } \\
\text { Income }\end{array}$ \\
\hline \multicolumn{6}{|l|}{$\begin{array}{l}\text { Equities: } \\
\text { Mutual Funds }\end{array}$} \\
\hline Equity Funds & $1,267,844$ & $1,710,288$ & 78.5 & 1.9 & 32,781 \\
\hline \multirow{4}{*}{$\begin{array}{l}\text { Fixed Income: } \\
\text { GNMA } \\
\text { Certificates of Deposit }\end{array}$} & & & & & \\
\hline & 22,063 & 25,709 & 1.2 & 10.8 & 2,775 \\
\hline & 5,000 & 5,000 & 0.2 & 8.3 & 415 \\
\hline & 27,063 & 30,709 & 1.4 & 10.4 & 3,190 \\
\hline \multirow{2}{*}{$\begin{array}{l}\text { Cash \& Equivalents: } \\
\text { Cash and Equivalents } \\
\text { Total Portfolio }\end{array}$} & 437,011 & 437,011 & 20.1 & 5.1 & 22,288 \\
\hline & $1,731,918$ & $2,178,008$ & $\overline{100.0}$ & 2.7 & 58,258 \\
\hline
\end{tabular}

TABLE 1.

APSA Balance Sheet

\begin{tabular}{|c|c|c|c|c|c|c|}
\hline & \multicolumn{3}{|c|}{ June 30,1992} & \multicolumn{3}{|c|}{ Total All Funds, June 30} \\
\hline & $\begin{array}{l}\text { General } \\
\text { Operating } \\
\text { Fund }\end{array}$ & $\begin{array}{l}\text { Trust and } \\
\text { Development } \\
\text { Fund }\end{array}$ & $\begin{array}{l}\text { Endowed } \\
\text { Program } \\
\text { Funds }\end{array}$ & 1992 & 1991 & 1990 \\
\hline $\begin{array}{l}\text { Assets: } \\
\text { Current } \\
\text { Property and Equipment }\end{array}$ & $\begin{array}{r}\$ 1,186,678 \\
441,890\end{array}$ & $\$ 1,715,440$ & $\$ 5,781,015$ & $\begin{array}{r}\$ 8,683,133 \\
441,890\end{array}$ & $\begin{array}{r}\$ 8,232,618 \\
453,087\end{array}$ & $\begin{array}{r}\$ 3,101,902 \\
502,963\end{array}$ \\
\hline $\begin{array}{l}\text { Liabilities and Fund Balance } \\
\text { Current } \\
\text { Fund Balances }\end{array}$ & $\begin{array}{r}554,663 \\
1,073,905\end{array}$ & $1,715,440$ & $5,781,015$ & $\begin{array}{r}554,663 \\
8,570,360\end{array}$ & $\begin{array}{r}524,890 \\
8,160,815\end{array}$ & $\begin{array}{r}573,029 \\
3,031,835\end{array}$ \\
\hline Total & $\$ 1,628,568$ & $\$ 1,715,440$ & $\$ 5,781,015$ & $\$ 9,125,023$ & $\$ 8,685,705$ & $\$ 3,604,684$ \\
\hline
\end{tabular}


ment and sound investments. Figures for the Trust and Development Fund are presented in Table 3. Data are provided for cost and market value and estimated annual income. The market value of the Trust and Development Fund stood at $\$ 2,178,008$ on June 30, 1992. For the fiscal year ending June 30,1992 , the fund as a whole grew at $8.74 \%$. The equity portion alone grew at $11.77 \%$.

Table 4 presents similar data for the Congressional Fellowship Program, endowed by $\mathrm{MCI}$. The market value of these funds was $\$ 5,787,659$ as of June 30, 1992. These funds grew at $16.1 \%$ for the fiscal year ending June 30, 1992.

Since becoming treasurer, many people have asked me two simple questions: "On what does the APSA spend its money and what are its major revenue sources?" While a full answer to this question would be too detailed to present here, Table 5 (Revenue Trends, 1987-92) and Table 6 (Expenditure Trends, 1986-92) provide an overview of the main categories of revenues and expenditures. Individual membership dues, institutional memberships, and advertising accounted for $60 \%$ of revenues for 1991-92. On the expenditure side, publications, special programs, and general operating expenditures for APSA programs and activities accounted for almost $75 \%$ of total expenses. Table 7 lists the salary scale for the national office.

The APSA Council took several actions at the recent meetings in Chicago (September 1-5, 1992). Because of the weak state of the economy, and the inability to rent out office space at the APSA headquarters, the budget figures for 1992-93 have been revised downward. These revisions were approved at the Council meeting on September 2, 1992. At the same time the Council authorized the establishment of an ad hoc Program Review Committee to expend up to $\$ 3,000$ for one meeting.

With these revisions the Association has projected a budget deficit of $\$ 34,310$ for the $1992-93$ year. To cover this anticipated deficit, a motion was made to move $\$ 55,000$ from the reserve fund $(\$ 55,000$ being the expected shortfall due to not renting space). This motion passed.

In addition, on the advice of our auditors, Maffey, Good, and Associ-
TABLE 4.

Portfolio Summary, Congressional Fellowship Program-Endowed, June 30, 1992

\begin{tabular}{|c|c|c|c|c|c|}
\hline Security Type & Cost Value & Market Value & $\begin{array}{c}\% \\
\text { Assets }\end{array}$ & $\begin{array}{l}\text { Current } \\
\text { Yield }\end{array}$ & $\begin{array}{c}\text { Estimated } \\
\text { Annual } \\
\text { Income }\end{array}$ \\
\hline \multicolumn{6}{|l|}{$\begin{array}{l}\text { Equities: } \\
\text { Mutual Funds }\end{array}$} \\
\hline Equity Funds & $4,200,000$ & $4,798,790$ & 82.9 & 1.3 & 61,164 \\
\hline \multirow[t]{2}{*}{ Bond Funds } & 800,000 & 913,211 & 15.8 & 7.9 & 71,966 \\
\hline & $5,000,000$ & $5,712,002$ & 98.7 & 2.3 & 133,130 \\
\hline $\begin{array}{l}\text { Cash \& Equivalents: } \\
\text { Cash and Equivalents }\end{array}$ & 75,657 & 75,657 & 1.3 & 5.5 & 4,160 \\
\hline Total Portfolio & $5,075,657$ & $5,787,659$ & $\overline{100.0}$ & 2.4 & 137,290 \\
\hline
\end{tabular}

TABLE 5.

Revenue Trends, 1987-1992

\begin{tabular}{|c|c|c|c|c|c|}
\hline & $1987-88$ & $1988-89$ & $1989-90$ & $1990-91$ & $1991-92$ \\
\hline Individual Membership & $\$ 432,902$ & $\$ 488,721$ & $\$ 501,486$ & $\$ 650,434$ & $\$ 667,320$ \\
\hline Institutional Membership & 301,359 & 303,809 & 305,345 & 380,996 & 395,972 \\
\hline Administrative & 89,599 & 74,514 & 59,158 & 61,281 & 73,772 \\
\hline Annual Meeting & 87,565 & 134,351 & 109,670 & 143,585 & 201,720 \\
\hline Advertising & 217,431 & 256,164 & 263,355 & 280,197 & 296,648 \\
\hline Dividends/Interest & 191,157 & 184,872 & 201,180 & 101,185 & 103,709 \\
\hline Sales & 59,172 & 74,853 & 102,215 & 138,208 & 130,697 \\
\hline Departmental Services & 91,382 & 145,281 & 144,819 & 190,146 & 153,405 \\
\hline Other & 167,070 & 184,587 & 204,545 & 212,076 & 231,601 \\
\hline Total & $\$ 1,637,637$ & $\$ 1,847,152$ & $\$ 1,891,773$ & $\$ 2,158,118$ & $\$ 2,254,844$ \\
\hline
\end{tabular}

TABLE 6.

Expenditure Trends, 1986-92

\begin{tabular}{|c|c|c|c|c|c|}
\hline & $1987-88$ & $1988-89$ & $1989-90$ & $1990-91$ & 1991-92 \\
\hline Publications & \$ 331,685 & \$ 371,627 & \$ 399,375 & $\$ 401,033$ & $\$ 438,286$ \\
\hline Annual Meeting & 112,573 & 125,459 & 148,708 & 204,639 & 189,970 \\
\hline Special Programs & 221,291 & 274,492 & 260,381 & 320,639 & 319,993 \\
\hline Governing the Association & 66,212 & 66,366 & 68,960 & 60,930 & 68,443 \\
\hline \multicolumn{6}{|l|}{ Membership, Business } \\
\hline Office and Sales & 184,372 & 215,704 & 257,407 & 292,561 & 294,566 \\
\hline General Operating & 647,119 & 644,485 & 736,474 & 853,722 & 869,286 \\
\hline \multicolumn{6}{|l|}{ IPSA Net Expenses Over } \\
\hline Income & - & 33,118 & - & - & - \\
\hline Total & $\$ 1,563,252$ & $\$ 1,731,251$ & $\$ 1,871,305$ & $\$ 2,133,524$ & $\$ 2,180,544$ \\
\hline
\end{tabular}

TABLE 7.

APSA National Office Salary Scale, 1992-93

\begin{tabular}{|c|c|c|c|c|c|c|}
\hline \multirow[b]{2}{*}{ APSA Position } & \multirow{2}{*}{$\begin{array}{l}\text { Equivalent } \\
\text { Government } \\
\text { Grade }\end{array}$} & \multirow{2}{*}{$\begin{array}{c}1992 \\
\text { Federal } \\
\text { Salary Scale }\end{array}$} & \multirow{2}{*}{$\begin{array}{l}1992-93 \\
\text { APSA } \\
\text { Grade }\end{array}$} & \multirow{2}{*}{$\begin{array}{c}1992-93 \\
\text { APSA } \\
\text { Salary Scale }\end{array}$} & \multicolumn{2}{|c|}{$\begin{array}{l}\text { Number of } \\
\text { Employees }\end{array}$} \\
\hline & & & & & FT & PT \\
\hline Political Scientists: & & & & & & \\
\hline Executive Director & $17-18$ & $86,519-101,404$ & 8 & $70,000-95,000$ & 1 & \\
\hline Deputy Director & $15-17$ & $64,233-98,056$ & 7 & $60,000-85,000$ & 1 & \\
\hline Program Directors & $13-15$ & $46,210-83,502$ & 6 & $40,000-75,000$ & 2 & 1 \\
\hline $\begin{array}{l}\text { Non-Political Scientists: } \\
\text { Program Managers } \\
\text { Senior Administrative }\end{array}$ & $11-12$ & $32,423-50,516$ & 5 & $30,000-40,000$ & 4 & \\
\hline Assistant & $9-10$ & $26,796-38,367$ & 4 & $20,000-35,000$ & 4 & \\
\hline Administrative Assistant & $6-8$ & $19,713-31,543$ & 3 & $18,000-30,000$ & 5 & \\
\hline $\begin{array}{l}\text { Secretary, Receptionist, } \\
\text { Maintenance }\end{array}$ & $4-7$ & $15,808-28,476$ & 2 & $15,000-27,000$ & 3 & \\
\hline Clerk & $1-3$ & $11,478-18,303$ & 1 & $11,000-17,000$ & 1 & 1 \\
\hline
\end{tabular}


ates, a motion was made to treat "interests and dividends" in Article VII, 9 of APSA's Constitution as part of the meaning of "investment income." According to our auditors, this is a reasonable interpretation of the phrase "interest and dividends." This motion passed.

Finally, the APSA has decided to move to a program budget, making it easier to see exactly what each program costs and the revenues it generates. Table 8 presents APSA's budget as it has been prepared previously and permits comparison with prior budget years, while Table 9 shows the same budget presented more programmatically. To calculate the true cost of a program, it is necessary to add $32 \%$ to cover each program's share of overall administration of the Association. So, for example, the direct cost of endowed awards is $\$ 22,558$, but the total cost of that program is $32 \%$ more, or $\$ 29,777$. We hope this new budget format will be easier to read and understand.
TABLE 8.

The APSA Traditional Budget

\begin{tabular}{|c|c|c|c|c|c|}
\hline & $\begin{array}{l}\text { Budget } \\
1990-91\end{array}$ & $\begin{array}{r}\text { Actual } \\
1990-91\end{array}$ & $\begin{array}{l}\text { Budget } \\
1991-92\end{array}$ & $\begin{array}{r}\text { Actual } \\
1991-92\end{array}$ & $\begin{array}{l}\text { Budget } \\
1992-93\end{array}$ \\
\hline \multicolumn{6}{|l|}{ REVENUE } \\
\hline $\begin{array}{l}\text { Membership } \\
\text { Professional } \\
\text { Student } \\
\text { Family } \\
\text { Life } \\
\text { Associate } \\
\text { Institutional }\end{array}$ & $\begin{array}{r}520,000 \\
90,000 \\
2,000 \\
2,000 \\
8,000 \\
380,000\end{array}$ & $\begin{array}{r}553,905 \\
85,038 \\
1,875 \\
1,700 \\
7,916 \\
380,996\end{array}$ & $\begin{array}{r}576,108 \\
88,400 \\
2,000 \\
1,000 \\
8,000 \\
385,000\end{array}$ & $\begin{array}{r}563,006 \\
94,309 \\
1,840 \\
1,300 \\
6,865 \\
395,972\end{array}$ & $\begin{array}{r}576,000 \\
98,000 \\
2,000 \\
1,000 \\
8,000 \\
410,000\end{array}$ \\
\hline Total Membership & $1,002,000$ & $1,031,430$ & $1,060,508$ & $1,063,292$ & $1,095,000$ \\
\hline Administrative & 80,000 & 61,281 & 60,000 & 73,772 & 50,000 \\
\hline Annual Meeting & 127,000 & 143,585 & 150,000 & 201,720 & 207,000 \\
\hline $\begin{array}{l}\text { Advertising } \\
\text { Review } \\
\text { PS } \\
\text { Program } \\
\text { Exhibits } \\
\text { Political Science Teacher }\end{array}$ & $\begin{array}{r}101,000 \\
14,000 \\
57,000 \\
100,000 \\
2,000\end{array}$ & $\begin{array}{r}100,437 \\
8,827 \\
62,846 \\
107,141 \\
946\end{array}$ & $\begin{array}{r}110,000 \\
14,000 \\
62,000 \\
115,000 \\
0\end{array}$ & $\begin{array}{r}104,253 \\
12,722 \\
61,898 \\
117,775 \\
-\end{array}$ & $\begin{array}{r}110,000 \\
15,000 \\
72,000 \\
124,000 \\
--\end{array}$ \\
\hline Total Advertising & 274,000 & 280,197 & 301,000 & 296,648 & 321,000 \\
\hline Dividends/Interest & 200,000 & 101,185 & 170,000 & 103,709 & 200,000 \\
\hline $\begin{array}{l}\text { Sales } \\
\text { Back Issues } \\
\text { Mailing Lists } \\
\text { Panel Paper, JDB, Other } \\
\text { Reprints } \\
\text { Minority Identification } \\
\text { APSA Publications }\end{array}$ & $\begin{array}{r}3,000 \\
60,000 \\
22,000 \\
4,000 \\
0 \\
23,000\end{array}$ & $\begin{array}{r}1,019 \\
66,761 \\
28,399 \\
3,837 \\
1,740 \\
36,452\end{array}$ & $\begin{array}{r}2,000 \\
70,000 \\
25,000 \\
4,000 \\
1,000 \\
17,500\end{array}$ & $\begin{array}{r}2,223 \\
66,610 \\
25,514 \\
4,956 \\
1,804 \\
29,590\end{array}$ & $\begin{array}{r}2,000 \\
70,000 \\
25,000 \\
4,000 \\
2,000 \\
45,000\end{array}$ \\
\hline Total Sales & 112,000 & 138,208 & 119,500 & 130,697 & 148,000 \\
\hline Departmental Services & 150,000 & 190,146 & 167,150 & 153,405 & 160,000 \\
\hline Rent & 100,800 & 100,217 & 110,000 & 107,693 & 55,000 \\
\hline Personnel Placement & 65,000 & 67,802 & 68,000 & 77,980 & 86,000 \\
\hline Section Dues & 30,000 & 28,226 & 30,000 & 30,363 & 32,000 \\
\hline Royalties & 10,000 & 14,403 & 14,000 & 13,349 & 14,000 \\
\hline Miscellaneous & 3,000 & 1,438 & 2,000 & 2,216 & 2,000 \\
\hline TOTAL REVENUE & $2,153,800$ & $2,158,118$ & $2,252,158$ & $2,254,844$ & $2,370,000$ \\
\hline
\end{tabular}

\section{EXPENSES}

\begin{tabular}{lrrrrr} 
Publications & & & & \\
Review & 185,000 & 167,592 & 176,000 & 168,009 & 167,200 \\
Printing & 30,000 & 28,800 & 36,000 & 33,089 & 34,800 \\
Postage & 80,000 & 73,326 & 80,000 & 86,172 & 84,000 \\
Salaries & 20,000 & 14,116 & 17,000 & 18,956 & 19,000 \\
Office and Editorial & & & 8,000 & 8,444 & \\
Transition & 315,000 & 283,834 & 317,000 & 314,670 & 305,000 \\
Total APSR & & & & & \\
PS & 85,000 & 85,255 & 90,000 & 93,690 & 100,000 \\
Printing & 24,000 & 23,046 & 30,000 & 27,863 & 28,000 \\
Postage & 3,000 & 1,288 & 3,000 & 2,064 & 3,000 \\
$\quad$ Editorial and Miscellaneous & 112,000 & 109,589 & 123,000 & 123,617 & 131,000 \\
$\quad$ Total PS & 10,000 & 7,610 & & 0 & \\
Political Science Teacher & 437,000 & 401,033 & 440,000 & 438,287 & 436,000 \\
Total Publications & & & & & \\
Annual Meeting & 30,000 & 29,858 & 30,000 & 25,750 & 30,000 \\
Printing & 7,500 & 7,737 & 7,500 & 3,435 & 7,000 \\
Program Committee & 80,000 & 98,887 & 60,000 & 74,105 & 90,000 \\
On-Site Expenses & 68,000 & 60,658 & 71,400 & 76,590 & 80,000 \\
Salaries-APSA & 5,000 & 7,499 & 6,000 & 10,091 & 10,000 \\
Exhibits & 190,500 & 204,639 & 174,900 & 189,971 & 217,000 \\
Total Annual Meeting & & & & & \\
Special Programs & & & & & \\
Committees & 5,000 & 5,532 & 5,000 & 5,018 & 5,000 \\
Status of Blacks & & & & & \\
\hline
\end{tabular}


TABLE 8 (continued)

\begin{tabular}{|c|c|c|c|c|c|}
\hline & $\begin{array}{c}\text { Budget } \\
1990-91\end{array}$ & $\begin{array}{r}\text { Actual } \\
1990-91\end{array}$ & $\begin{array}{l}\text { Budget } \\
1991-92\end{array}$ & $\begin{array}{r}\text { Actual } \\
1991-92\end{array}$ & $\begin{array}{l}\text { Budget } \\
1992-93\end{array}$ \\
\hline Status of Latinos & 3,000 & 5,551 & 5,000 & 4,699 & 5,000 \\
\hline Status of Women & 3,000 & 2,723 & 3,000 & 1,356 & 3,000 \\
\hline Education & 3,000 & 2,806 & 3,000 & 3,069 & 3,000 \\
\hline Ethics, Rights, Freedom & 5,000 & 6,766 & 5,000 & 6,712 & 7,000 \\
\hline Research Support & 3,000 & 2,513 & 3,000 & 1,796 & 3,000 \\
\hline Publications & 3,000 & 2,754 & 3,000 & 3,723 & 3,000 \\
\hline International Political Science & 3,000 & 1,343 & 3,000 & 767 & 3,000 \\
\hline Endowed Programs & 3,000 & 290 & 3,000 & 10 & 3,000 \\
\hline Ad Hoc Ethics Charter & 2,000 & 1,383 & 0 & 0 & \\
\hline APSR Editor Search & 0 & & & & \\
\hline Status of Lesbians and Gays & 0 & & 3,000 & 2,276 & 5,000 \\
\hline Task Force on Rule of Three & 0 & & & & 1,500 \\
\hline $\begin{array}{l}\text { Organized Sections } \\
\text { Ad Hoc Program Review }\end{array}$ & 0 & & & & 1,500 \\
\hline Committee & & & & & 3,000 \\
\hline Total Committees & 33,000 & 31,661 & 36,000 & 29,426 & 46,000 \\
\hline Placement Service & & & & & \\
\hline Printing and Postage & 27,000 & 23,443 & 28,000 & 27,542 & 29,500 \\
\hline Salaries-APSA & 35,000 & 36,179 & 38,000 & 38,779 & 40,500 \\
\hline Total Placement Service & 62,000 & 59,622 & 66,000 & 66,321 & 70,000 \\
\hline Black Fellowship & 6,000 & 6,000 & 6,000 & 6,000 & 6,000 \\
\hline Latino Fellowship & 6,000 & & 6,000 & & 6,000 \\
\hline Membership Constituent Society & 37,528 & 37,365 & 39,407 & 36,951 & 40,000 \\
\hline Departmental Services & 145,500 & 138,801 & 146,500 & 143,839 & 160,000 \\
\hline Education & 16,000 & 13,713 & 20,000 & 14,328 & 20,000 \\
\hline Oral History Project & 2,000 & 2,000 & 2,000 & 128 & 0 \\
\hline Small Research Grants & 15,000 & 15,457 & 15,000 & 14,999 & 15,000 \\
\hline International Travel Grants & 8,000 & 7,965 & 8,000 & 8,000 & 8,000 \\
\hline Total Special Programs & 331,028 & 312,584 & 344,907 & 319,992 & 371,000 \\
\hline \multicolumn{6}{|l|}{ Governing the Association } \\
\hline Council & 25,000 & 22,581 & 25,000 & 24,461 & 26,500 \\
\hline Elections & 5,000 & & 5,000 & & 5,000 \\
\hline Administrative Committee & 4,500 & 6,260 & 6,500 & 6,369 & 6,000 \\
\hline Accounting-Legal Fees & 22,000 & 17,219 & 20,000 & 20,681 & 25,000 \\
\hline Presidential & 5,000 & 3,141 & 5,000 & 5,893 & 6,000 \\
\hline Nominating Committee & 3,500 & 4,199 & 4,000 & 3,109 & 4,000 \\
\hline Elections Committee & 500 & & 500 & & 500 \\
\hline Professional Liability Insurance & 6,500 & 7,530 & 8,000 & 7,930 & 10,500 \\
\hline Total Governing the Association & 72,000 & 60,930 & 74,000 & 68,443 & 83,500 \\
\hline \multicolumn{6}{|l|}{$\begin{array}{l}\text { Membership, Business Office, } \\
\text { and Cost of Sales }\end{array}$} \\
\hline Membership & & & & & \\
\hline $\begin{array}{l}\text { Recruiting and Supplies } \\
\text { Salaries-APSA }\end{array}$ & $\begin{array}{r}22,000 \\
114,000\end{array}$ & $\begin{array}{r}23,555 \\
114,615\end{array}$ & $\begin{array}{r}15,000 \\
119,700\end{array}$ & $\begin{array}{r}17,789 \\
121,325\end{array}$ & $\begin{array}{r}20,000 \\
127,000\end{array}$ \\
\hline Total Membership & 136,000 & 138,170 & 134,700 & 139,114 & 147,000 \\
\hline Business Office Salaries-APSA & 81,500 & 84,336 & 87,000 & 95,215 & 95,000 \\
\hline \multicolumn{6}{|l|}{ Cost of Sales } \\
\hline Printing, Fees, Publishing & 27,000 & 22,265 & 27,000 & 14,855 & 15,000 \\
\hline COGS APSA Publishing & 0 & 13,043 & & 3,627 & 29,000 \\
\hline Salaries-APSA & 38,823 & 38,220 & 41,500 & 39,910 & 43,990 \\
\hline Instructional Monographs & 7,000 & 4,582 & 1,000 & 1,371 & 1,500 \\
\hline COGS Monographs/Project '87 & & & 500 & 475 & 1,000 \\
\hline Total Cost of Sales & 72,823 & 78,110 & 70,000 & 60,238 & 90,490 \\
\hline $\begin{array}{l}\text { Total Membership, Business } \\
\text { Office, Cost of Sales }\end{array}$ & 290323 & 300 & 201 & $?$ & \\
\hline \multicolumn{6}{|l|}{ General Operating } \\
\hline Salaries-APSA & 479,000 & 478,664 & 485,000 & 493,005 & 550,000 \\
\hline \multicolumn{6}{|l|}{ Building } \\
\hline Taxes & 60,799 & 69,811 & 74,000 & 71,595 & 74,000 \\
\hline Repair and Upkeep & 8,759 & 7,966 & 10,000 & 10,362 & 10,000 \\
\hline Utilities & 21,431 & 23,493 & 25,000 & 23,138 & 25,000 \\
\hline Supplies & 9,496 & 4,777 & 7,000 & 5,513 & 7,000 \\
\hline
\end{tabular}


TABLE 8 (continued)

\begin{tabular}{|c|c|c|c|c|c|}
\hline & $\begin{array}{l}\text { Budget } \\
1990-91\end{array}$ & $\begin{array}{r}\text { Actual } \\
1990-91\end{array}$ & $\begin{array}{l}\text { Budget } \\
1991-92\end{array}$ & $\begin{array}{r}\text { Actual } \\
1991-92\end{array}$ & $\begin{array}{l}\text { Budget } \\
\text { 1992-93 }\end{array}$ \\
\hline Insurance & 7,101 & 8,270 & 10,000 & 11,891 & 12,000 \\
\hline Depreciation & 11,787 & 13,545 & 13,500 & 12,990 & 18,000 \\
\hline Custodial Salaries & 38,093 & 39,252 & 42,000 & 42,291 & 44,520 \\
\hline Total Building & 157,466 & 167,114 & 181,500 & 177,780 & 190,520 \\
\hline \multicolumn{6}{|l|}{$\begin{array}{l}\text { Equipment, Supplies and } \\
\text { Administrative }\end{array}$} \\
\hline Computer Supplies and Services & & & 10,000 & 11,499 & 12,000 \\
\hline Postage & 42,786 & 43,751 & 52,500 & 39,957 & 41,650 \\
\hline Printing Stationery & 11,515 & 13,964 & 14,000 & 14,651 & 14,000 \\
\hline Mailing Services and Storage & 13,618 & 17,580 & 18,000 & 14,964 & 16,650 \\
\hline Telephone, Fax & 12,415 & 14,268 & 15,000 & 14,512 & 15,000 \\
\hline Office Supplies & 10,760 & 13,100 & 12,000 & 11,312 & 12,000 \\
\hline Staff Travel & 6,999 & 6,940 & 7,500 & 9,291 & 8,000 \\
\hline Miscellaneous & 9,196 & 5,936 & 5,000 & 5,281 & 5,500 \\
\hline Administrative & 7,212 & 6,564 & 6,000 & 3,682 & 6,000 \\
\hline Payroll Services & & & 1,000 & 1,259 & 1,000 \\
\hline Insurance & 5,217 & 4,539 & 8,000 & 5,906 & 7,000 \\
\hline Miscellaneous Bank Charges & & 6,765 & 7,000 & 8,667 & 9,000 \\
\hline Depreciation & 36,271 & 47,327 & 50,000 & 29,076 & 40,000 \\
\hline Maintenance/Repair & 24,907 & 24,886 & 28,000 & 23,065 & 28,000 \\
\hline Annual Leave & 5,473 & 2,324 & 7,000 & 5,377 & 7,000 \\
\hline $\begin{array}{l}\text { Total Equipment, Supplies, } \\
\text { Administration }\end{array}$ & 186,369 & 207,944 & 241,000 & 198,499 & 222,800 \\
\hline Total General Operating & 822,835 & 853,722 & 907,500 & 869,284 & 963,320 \\
\hline $\begin{array}{l}\text { TOTAL EXPENDITURES } \\
\text { EXPENSE OVER REVENUE }\end{array}$ & $\begin{array}{r}2,143,686 \\
0\end{array}$ & $\begin{array}{r}2,133,524 \\
0\end{array}$ & $2,233,007$ & $2,180,544$ & $\begin{array}{r}2,403,310 \\
(33,310)\end{array}$ \\
\hline REVENUE OVER EXPENSE & 10,114 & 24,594 & 19,151 & 74,300 & 0 \\
\hline $\begin{array}{l}\text { LIFE MEMBERSHIP } \\
\text { RESERVE FUND }\end{array}$ & & & & $(1,300)$ & $\begin{array}{l}(1,000) \\
55,000\end{array}$ \\
\hline NET REVENUE & 10,114 & 24,594 & 19,151 & 73,000 & 20,690 \\
\hline
\end{tabular}

TABLE 9.

APSA Program Budget

\begin{tabular}{|c|c|c|c|c|c|}
\hline & $\begin{array}{r}\text { 1992-93 } \\
\text { Budget }\end{array}$ & & $\begin{array}{r}1992-93 \\
\text { Budget }\end{array}$ & & $\begin{array}{r}1992-93 \\
\text { Budget }\end{array}$ \\
\hline REVENUE & & Panel Papers, Program Sales & 20,000 & Style Manual & 3,000 \\
\hline Membership & & Child Care Registration & 1,500 & Project ' 87 and Monographs & 4,000 \\
\hline Individual & & Total Annual Meeting & 424,500 & Miscellaneous (S.S., Vote 88, & \\
\hline Professional & 548,500 & Retained Earnings ${ }^{b}$ & & $\begin{array}{l}\text { Camp 80) } \\
\text { Total APSA Publications }\end{array}$ & $\begin{array}{r}8,000 \\
45,000\end{array}$ \\
\hline Student & 98,000 & T \& D Fund & 109,000 & 1 Otal APSA PUDications & 5000 \\
\hline Family & 2,000 & General APSA Fund & 109,000 & Journal Discount Program & 5,000 \\
\hline Life & 1,000 & Endowed Program Funds/Awards & 10,275 & Total Sales and Advertising & 266,200 \\
\hline $\begin{array}{l}\text { Associate } \\
\text { Retired }\end{array}$ & $\begin{array}{r}8,000 \\
14.500\end{array}$ & Total Earnings & 228,275 & Employment Services & \\
\hline Joint/Other Associations & 1,000 & Sales and Advertising & & Personnel Placement Service & \\
\hline Total & 673,000 & Mailing Lists & 70,000 & Subscriptions & 56,000 \\
\hline $\begin{array}{l}\text { Institutional } \\
\text { Section Dues }\end{array}$ & 410,000 & & & $\begin{array}{l}\text { Advertising } \\
\text { Foreign Postage }\end{array}$ & $\begin{array}{r}24,000 \\
\cdot 1,500\end{array}$ \\
\hline $\begin{array}{l}\text { Section Dues } \\
\text { Foreign Postage }\end{array}$ & $\begin{array}{l}62,000 \\
12,000\end{array}$ & $\begin{array}{l}\text { Reprints for Authors } \\
\text { Back Issues }\end{array}$ & $\begin{array}{l}4,000 \\
1,800\end{array}$ & PSN Payment from DSP & 18,000 \\
\hline Total Membership & $1,157,000$ & Advertising & 110,000 & Total Personnel Placement & 99,500 \\
\hline Administrative $^{\mathrm{a}}$ & & $\begin{array}{l}\text { Permissions and Royalties } \\
\text { P }\end{array}$ & 12,000 & Credential Referral Service & \\
\hline $\begin{array}{l}\text { Wohlers } \\
\text { Ouest }\end{array}$ & 10,000 & Total APSR & 121,800 & Credential Referral Membership & 4,500 \\
\hline $\begin{array}{l}\text { Quest } \\
\text { CFP }\end{array}$ & 1,000 & $P S$ & & Total Credential Referral & 4,500 \\
\hline $\begin{array}{l}\text { CFP } \\
\text { Grants }\end{array}$ & 28,000 & Advertising & 15,000 & Total Employment Services & 104,000 \\
\hline $\begin{array}{l}\text { Grants } \\
\text { Total Administrative }\end{array}$ & 11,000 & $\begin{array}{l}\text { Back Issues } \\
\text { Permissions \& Royalties }\end{array}$ & $\begin{array}{r}200 \\
2.000\end{array}$ & Programs for Departments & \\
\hline $\begin{array}{l}\text { Total Administrative } \\
\text { Annual Meeting }\end{array}$ & 50,000 & $\begin{array}{l}\text { Permissions \& Royalties } \\
\text { Total PS }\end{array}$ & 17,200 & Departmental Services & \\
\hline Registration & 207,000 & Other Advertising & 1,200 & $\begin{array}{l}\text { Dues } \\
\text { Book Sales }\end{array}$ & $\begin{array}{r}115,000 \\
45,000\end{array}$ \\
\hline Exhibits & 124,000 & APSA Publications ${ }^{c}$ & & Total Departmental Services & 160,000 \\
\hline Program Advertising & 72,000 & State of the Discipline & 30,000 & & \\
\hline
\end{tabular}




\begin{tabular}{|c|c|c|c|c|c|}
\hline & $\begin{array}{r}\text { 1992-93 } \\
\text { Budget }\end{array}$ & & $\begin{array}{r}1992-93 \\
\text { Budget }\end{array}$ & & $\begin{array}{r}\text { 1992-93 } \\
\text { Budget }\end{array}$ \\
\hline Minority Identification & 2,000 & Support for Committees & & Support for Publications & \\
\hline Total Department Programs & 162,000 & Staff & 124,635 & Storage & 3,000 \\
\hline Rent & 55,000 & Supplies, Postage, Phone & 5,000 & Fulfillment & 5,000 \\
\hline Miscellaneous & 2,000 & Total Support & 129,635 & Advertising & 1,000 \\
\hline TOTAL REVENUE & $2,448,975$ & Total Committee Programs & 196,635 & $\begin{array}{l}\text { Permissions } \\
\text { Staff }\end{array}$ & $\begin{array}{r}5,000 \\
43,990\end{array}$ \\
\hline & & Employment Services & & Total Support & 57,990 \\
\hline EXPENSES & & $\begin{array}{l}\text { Personnel Placement Service } \\
\text { Printing }\end{array}$ & 15,500 & Total Publications & 605,328 \\
\hline Programs & & Postage & 22,000 & Governance & \\
\hline Annual Meeting & & Supplies & 1,500 & Council & \\
\hline Printing (Preliminary \& Final) & 30,000 & Staff & 25,000 & Meetings & 26,500 \\
\hline Exhibits & 10,000 & Annual Meeting & 10,500 & Administrative Committee & 6,000 \\
\hline Travel & 3,000 & Total Placement Service & 74,500 & Administration Elections & 5,500 \\
\hline On-site Personnel \& Services & 53,750 & Credential Referral Service & & Total Council & 38,000 \\
\hline $\begin{array}{l}\text { Program Committee } \\
\text { Staff }\end{array}$ & 7,000 & Postage & 5,000 & Presidential & 6,000 \\
\hline $\begin{array}{l}\text { Staff } \\
\text { Permanent }\end{array}$ & 99,250 & $\begin{array}{l}\text { Xeroxing } \\
\text { Supplies }\end{array}$ & $\begin{array}{l}3,500 \\
1,500\end{array}$ & Nominating Committee & 4,000 \\
\hline Temporary & 5,750 & Staff & 20,500 & Accounting and Legal & 25,000 \\
\hline $\begin{array}{l}\text { Supplies, Postage, Phones } \\
\text { Short Courses, Special Events }\end{array}$ & $\begin{array}{l}5,000 \\
1,000\end{array}$ & Total Credential Referral & 30,500 & Professional Liability Insurance & 10,500 \\
\hline Child Care & 6,000 & Total Employment Services & 105,000 & Staff & 50,034 \\
\hline Promotion & 7,000 & Education Program & & Administrative & 2,000 \\
\hline $\begin{array}{l}\text { International Travel Grants } \\
\text { Task Force on Rule of Three }\end{array}$ & $\begin{array}{l}8,000 \\
1,500\end{array}$ & $\begin{array}{l}\text { Committee Meetings } \\
\text { Staff }\end{array}$ & $\begin{array}{r}3,000 \\
42,990\end{array}$ & Total Governance & 135,534 \\
\hline Total Annual Meeting & 237,250 & Supplies, Phone, Postage & 1,500 & \multicolumn{2}{|l|}{ APSA Representation of the Profession } \\
\hline Departmental Programs & & Total Education & 47,490 & Membership in Constituent & \\
\hline Departmental Services & & APSA Endowed Awards & & $\begin{array}{l}\text { Societies }^{\mathrm{g}} \\
\text { NHA }\end{array}$ & \\
\hline Printing & 41,600 & $\begin{array}{l}\text { Cash Awards } \\
\text { Staff }\end{array}$ & $\begin{array}{r}8,250 \\
13,308\end{array}$ & $\begin{array}{l}\text { NHA } \\
\text { COSSA }\end{array}$ & $\begin{array}{r}3,750 \\
18,550\end{array}$ \\
\hline $\begin{array}{l}\text { Postage, Phones } \\
\text { Brochures, Letters }\end{array}$ & $\begin{array}{r}13,000 \\
8,000\end{array}$ & $\begin{array}{l}\text { Staff } \\
\text { Administrative }\end{array}$ & 1,000 & ACLS & 1,200 \\
\hline $\begin{array}{l}\text { Brochures, Letters } \\
\text { Survey Analysis }\end{array}$ & $\begin{array}{l}8,000 \\
8,000\end{array}$ & Total Endowed & 22,558 & SSRC & 0 \\
\hline DS Committee & 3,000 & Oral History Project & 0 & AAAS & 1,250 \\
\hline Staff & 64,900 & Organized Sections & & ACE & 950 \\
\hline PSN Payment & 18,000 & Rebate to Sections & 30,000 & IPSA & 8,300 \\
\hline Other & 3,500 & Staff & 23,618 & ASS & 0 \\
\hline Total DS & 160,000 & Membership Lists & 8,400 & COPAFS & 5,600 \\
\hline Minority ID & & Subventions & 1,000 & $\begin{array}{l}\text { NCCPH } \\
\text { CNSF }\end{array}$ & 400 \\
\hline Postage, Printing & 1,500 & Ad Hoc Task Force & 1,500 & $\begin{array}{l}\text { CNSF } \\
\text { \% Increase }\end{array}$ & 0 \\
\hline Staff & 16,128 & Total Organized Sections ${ }^{\mathrm{d}}$ & 64,518 & $\begin{array}{l}\% \text { Increase } \\
\text { Total Membership }\end{array}$ & 40,000 \\
\hline Total Minority ID & 17,628 & Total Membership Service & & Staff & 40,025 \\
\hline Total Departmental Program & 177,628 & \& Programs & 436,201 & Administrative & 750 \\
\hline Membership Services and Programs & & Total Programs & 851,079 & $\begin{array}{l}\text { Regional, State, International } \\
\text { Travel }\end{array}$ & \\
\hline Committee Programs & & Publications & & $\begin{array}{l}\text { Travel } \\
\text { Total Representation }\end{array}$ & 88,775 \\
\hline $\begin{array}{l}\text { Status of Blacks } \\
\text { Meetings }\end{array}$ & & $A P S R^{\mathrm{e}}$ & & Business Office \& & \\
\hline $\begin{array}{l}\text { Meetings } \\
\text { Fellowships }\end{array}$ & $\begin{array}{l}5,000 \\
6,000\end{array}$ & $\begin{array}{l}\text { Printing } \\
\text { Postage }\end{array}$ & $\begin{array}{r}167,200 \\
34,800\end{array}$ & Membership Office & \\
\hline Total COSBP & 11,000 & Operating Expense & 16,600 & Business Office & \\
\hline Status of Latinos & & Editorial Staff & 48,000 & Investment Services & 18,000 \\
\hline Meeting & 5,000 & Honoraria-Editors & 36,000 & Supplies \& Services & 5,500 \\
\hline Fellowship & 6,000 & Reprints by authors & 4,000 & Bank Charges & 9,000 \\
\hline Total COSLP & 11,000 & Editorial Expense & 2,400 & Staff & 97,000 \\
\hline Status of Women & 3,000 & Total $A P S R$ & 309,000 & $\begin{array}{l}\text { Payroll Services } \\
\text { Total Business Office }\end{array}$ & $\begin{array}{r}1,000 \\
130,500\end{array}$ \\
\hline Status of Lesbians and Gays & 5,000 & $\begin{array}{l}\text { PS/Teacher } \\
\text { Printing }\end{array}$ & & Membership, Subscription Fulfi & 130,500 \\
\hline Ethics, Rights \& Freedoms & 7,000 & $\begin{array}{l}\text { Printing } \\
\text { Postage }\end{array}$ & $\begin{array}{r}100,000 \\
28,000\end{array}$ & Mailing Lists & ent, \\
\hline Research Support & & Editorial & 3,000 & Supplies & 5,000 \\
\hline Meetings & 3,000 & Staff & 65,838 & Printing & 10,000 \\
\hline Research Grants & 15,000 & Total PS/Teacher & 196,838 & Postage & 5,200 \\
\hline Total Research & 18,000 & & & Staff & 127,000 \\
\hline International Political Science & & Other APSA Publications & & $\begin{array}{l}\text { Total Membership, Subscription } \\
\text { Fulfillment, Mailing Lists }\end{array}$ & 147 \\
\hline Meeting & 3,000 & Publicity-Flyers \& Brochures & 3,000 & Total Business Office & \\
\hline Endowed Programs & 3,000 & $\begin{array}{l}\text { Cost of Goods Sold-APSA }{ }^{f} \\
\text { Journal Discount Brochures }\end{array}$ & $\begin{array}{r}31,900 \\
4,100\end{array}$ & \& Membership & 277,700 \\
\hline Publications & 3,000 & COGS-Monographs \& & & General Administrative & \\
\hline Ad Hoc Program Review & & Project ' 87 & 2,500 & Postage \& Mailing Services & 37,250 \\
\hline Committee & 3,000 & Total Other Publications & 41,500 & Stationery, Envelopes & 12,000 \\
\hline
\end{tabular}


TABLE 9 (continued)

\begin{tabular}{|c|c|c|c|c|c|}
\hline & $\begin{array}{r}\text { 1992-93 } \\
\text { Budget }\end{array}$ & & $\begin{array}{r}\text { 1992-93 } \\
\text { Budget }\end{array}$ & & $\begin{array}{r}1992-93 \\
\text { Budget }\end{array}$ \\
\hline $\begin{array}{l}\text { Xerox } \\
\text { Telephone, Fax } \\
\text { Office and Computer Supplies } \\
\text { General Administrative } \\
\text { General Operating Staff } \\
\text { Miscellaneous } \\
\text { Computer Support } \\
\text { Fees } \\
\text { Staff } \\
\text { Insurance (Workmans' Comp., } \\
\text { Bonding, Travel) }\end{array}$ & $\begin{array}{r}1,000 \\
15,000 \\
12,650 \\
8,025 \\
95,306 \\
1,000 \\
\\
8,000 \\
61,118 \\
7,000\end{array}$ & $\begin{array}{l}\text { Total General Administrative } \\
\text { Building and Equipment } \\
\text { Taxes } \\
\text { Repair and Upkeep } \\
\text { Maintenance Contracts } \\
\text { Depreciation } \\
\text { Insurance } \\
\text { Utilities } \\
\text { Building Supplies } \\
\text { Custodial Staff } \\
\text { Total Building \& Equipment }\end{array}$ & $\begin{array}{r}74,000 \\
10,000 \\
28,000 \\
58,000 \\
12,000 \\
25,000 \\
7,000 \\
44,520 \\
258,520\end{array}$ & $\begin{array}{l}\text { Subtotal Expenses }^{\text {h }} \\
\text { Pending Obligations } \\
\text { Annual Leave } \\
\text { Life Memberships } \\
\text { Total Pending } \\
\text { TOTAL EXPENSES } \\
\text { Revenue over Expenses } \\
\text { Expenses over Revenue } \\
\text { Reserve Fund } \\
\text { NET REVENUE }\end{array}$ & $\begin{array}{r}7,000 \\
1,000 \\
8,000 \\
2,483,285 \\
\\
(34,310) \\
55,000 \\
20,690\end{array}$ \\
\hline
\end{tabular}

${ }^{a}$ Wohlers and Quest pay fees to APSA for offering insurance and travel services to members and for APSA's assistance in making these services available to members. The CFP (Congressional Fellowship Program) is treated as a grant program.

' Retained earnings constitute the amount of earnings on APSA's investments (the Trust and Development Fund, our general cash flow reserve, and award funds) that are put directly into APSA's operating budget in accordance with APSA's By-Laws, Council policy, and procedures established by the Trust and Development Committee.

'Not including publications sponsored by the Departmental Services Program.

${ }^{d}$ Additional expenses related to the operation of organized sections are incorporated in the following other parts of this budget: Annual Meeting, Membership, Business Office, PS, Governance, General Administration.

'These expenses do not include the in-kind contributions of the University of Rochester, including overhead, equipment, graduate assistants, and relief-time for the Managing Editor.

${ }^{\mathrm{f}}$ COGS (Cost of Goods Sold) is an accounting technique that charges the expense side of the budget for the value of the publications sold in the fiscal year. Unsold publications are listed as inventory and counted as a depreciating asset in our annual audit.

${ }^{8}$ National Humanities Alliance, Consortium of Social Science Associations, American Council of Learned Societies, Social Science Research Council, American Association for the Advancement of Science, American Council on Education, International Political Science Association, American Association for the Advancement of Slavic Studies, Council of Professional Association on Federal Statistics, National Coordinating Committee for the Promotion of History, Coalition for National Science Funding. APSA is a member of, but pays no dues to: SSRC, AAASS, and CNSF.

${ }^{\text {h}}$ The following programs have independent funding and budgets, and they are reflected in APSA's annual audit: Kirkpatrick Fund, Congressional Fellowship Program, Ralph Bunche Summer Institute, IBM Poliware, other outside grants (these vary from year to year and fund activities, APSA staff, and administrative expenses). FIPSE-Course Syllabi Collection Project, Pew Charitable Trusts-Bill of Rights Education Collaborative Faculty Workshops, Japan-U.S. Friendship Commission-Japan Workshops, MacArthur-South Africa Workshop, NSF Workshop on Data Analysis, NSF/Argonne National Laboratory-Global Warming Conference.

\section{Report of the Managing Editor of the American Political Science Review, 1991-92}

\section{G. Bingham Powell, Jr. University of Rochester}

Fiscal year 1991-92 was a time of transitions for the $A P S R$. This report is divided into sections corresponding to the three major transitions: to a new managing editor and new editorial staff in Rochester; to a new format and a new press; to a new Book Review editor and editorial staff in Austin.

\section{Transition of Managing Editors}

Ohio State to Rochester. Pat Patterson completed six years of outstand- ing service to the profession as managing editor of the $A P S R$ at the end of the summer of 1991. After careful discussion about the mechanics of transition, we arranged a several-stage transfer of responsibility from Pat and his staff at Ohio State to the new editorial team in Rochester. The general concept was for Pat and Ohio State to continue editing all the 1991 issues and turn over responsibility to Bing Powell and Rochester for the March 1992 issue.

After the December 1991 issue was filled, in late June 1991, Pat began to send all manuscripts and referee reports that were not obvious rejects, to Rochester for final decision (accept, revise and resubmit, reject). These were manuscripts that would be the basis for the March 1992 and subsequent issues. Ohio State continued through the summer to reject manuscripts where the referees clearly advised against publication, largely a mechanical task. Ohio State also continued to receive all new manuscripts and assign them to referees through the summer. After about August 15 new manuscripts were sent to Rochester, where the new editorial team was being assembled. Ohio State also continued through the fall semester all the work involving the December 1991 issue, including the copy-editing and handling of galley proofs, page proofs, and blue lines. Michael Lane, who has been copyediting the Review for the past five years at Ohio State, continued as copy editor for the new Rochester team for the 1992 issues.

In general the transition procedure worked very well. I want to express my appreciation to Pat Patterson for arranging a transition that allowed 